\title{
The suprafascial course of lower leg perforators: An anatomical study
}

\author{
Luca Vaienti ${ }^{1}$, Giuseppe Cottone ${ }^{2}$, Francesco De Francesco ${ }^{1}$, Francesco Borelli ${ }^{1}$, \\ Giovanna Zaccaria ${ }^{3}$, Francesco Amendola ${ }^{1}$ \\ ${ }^{1}$ Department of Plastic and Reconstructive Surgery, I.R.C.C.S. Policlinico San Donato, San Donato Milanese; ${ }^{2}$ Department of Reconstructive \\ Surgery and Hand Surgery, AOU Ospedali Riuniti, Ancona; ${ }^{3}$ Department of Plastic and Reconstructive Surgery, University Hospital of \\ Modena, Modena, Italy
}

Background Perforator mapping has been well described in the literature. Once the suprafascial plane is reached, the course of perforators is considered constant. However, the surgeon must be aware of whether an anastomosis exists between perforators superficially to the fascia, in order to choose the best vessel upon which to base the reconstruction. Our retrospective in vivo anatomical study of lower leg perforator flaps presents the first description of variations in the suprafascial path of perforators, which may influence preoperative flap design.

Methods An anatomical study of lower limb perforators was performed on 46 nonconsecutive patients who were referred to our department from June 2012 to October 2018. Reconstruction with perforator-based propeller flaps was planned for each of the patients. In total, 72 perforators were preoperatively identified and surgically isolated. The suprafascial course of each perforator was reported.

Results During suprafascial surgical exploration, branching patterns were observed in four perforators. These perforators had been classified as single vessels in the preoperative ultrasonographic analysis. However, after surgical dissection, distal converging branches were noted in two of them.

Conclusions Our study is the first description in the literature of suprafascial converging perforators, which might constitute an obstacle to planned reconstruction procedures. Despite the accuracy of preoperative evaluations, anatomical variations were present. Knowledge of suprafascial perforator variations may help surgeons to choose the correct perforator upon which to base a planned flap.

Keywords Arteries / Lower extremity / Perforator flaps

\author{
Correspondence: \\ Francesco Amendola \\ Department of Plastic and \\ Reconstructive Surgery, I.R.C.C.S. \\ Policlinico San Donato, Piazza \\ Edmondo Malan 2, San Donato \\ Milanese 20097, Italy \\ Tel: $+39-252774458$ \\ Fax: $+39-252774459$ \\ E-mail: fgsamendola@gmail.com
}

Received: July 12, 2019 - Revised: February 12, 2020 - Accepted: February 13, 2020

pISSN: 2234-6163 • elSSN: 2234-6171 • https://doi.org/10.5999/aps.2019.00962 • Arch Plast Surg 2020;47:165-170

\section{INTRODUCTION}

Extensive soft tissue defects of the lower leg, especially in the distal third, are challenging for surgeons [1-3]. Local perforators
[4] or distant free flaps are often required to achieve full reconstruction.

According to the definition of the Gent International Course on Perforator Flaps [5], a perforator flap is composed of skin 
and subcutaneous tissue, nourished by perforator vessels arising from deep vascular system. Running through muscles or between muscular septa [5], a perforator pierces the fascia to provide a specific blood supply [5-7] to the overlying skin.

Perforator flaps in the lower leg mainly originate from the tibialis posterior and peroneal arteries [8]. Preoperative planning to identify perforator vessels is performed via Doppler ultrasonography or computed tomography angiography [9-14]. Perforator flaps are designed based on the preoperative imaging results [15]. The subfascial course and points of fascial perforation of the vessel are well identified by both of the abovementioned imaging techniques. Nonetheless, the suprafascial course of perforators is poorly defined.

Our study describes unusual anatomical variations in the suprafascial course of perforators. Some very close emerging perforators converged in a single common branch distally. When the dissection was limited to the immediate suprafascial plane, only one of those perforators could be preserved. However, extending the dissection further distally, we encountered a common converging branch. Once it is single-pedicled, the flap can be inset without risk of kinking. Thus, a more accurate dissection may avoid reducing the native vasculature of the flap.

\section{METHODS}

We retrospectively evaluated 46 nonconsecutive patients requiring lower leg reconstruction with local perforator flaps, from both the tibialis posterior and peroneal arteries, who were referred to our department from June 2012 to October 2018. The ages of the patients ranged from 25 to 65 years (mean, $46 \pm 11$ years). In total, 30 male and 16 female patients were included. The location of perforators was preoperatively identified using a Dopplex DMX device with a VP10 XS 10-MHz probe (Huntleigh Healthcare Ltd., Cardiff, UK).

All the patients were evaluated and treated by the same surgeon (LV). The emerging sites of cutaneous perforators traced using the Doppler ultrasonography were marked preoperatively. The flap area and contour were then defined, and dissection was initiated. Flap dissection was performed under loupe magnification, under general anaesthesia, and with a tourniquet appliedbut not inflated-at the base of the thigh. To raise a flap based on a posterior tibial artery perforator, the patient is placed in a supine position and the leg is slightly abducted and externally rotated. To raise a flap based on a peroneal artery perforator, the patient is also in the supine position, with a sandbag placed under the hip, which is flexed and internally rotated. We made generous incisions, and the approach to the pedicle was always suprafascial.
Sharp suprafascial dissection was carried out, directed toward the point where the perforator had been preoperatively identified. During the dissection, all other perforators encountered were isolated on a Silastic band and preserved. Once all the perforators in the region were isolated, we consecutively clamped each of them, to better understand which was the most reliable. A fasciocutaneous island flap was then based on the chosen perforator and rotated to cover the defect.

Different perforator vascular patterns were retrospectively collected, based on intraoperative photographic material previously obtained with explicit consent of the patients. Vascular patterns were then compared with the preoperative ultrasound results. The characteristics of the patients and the procedures are presented in Table 1.

\section{RESULTS}

In total, 72 cutaneous perforators ( 38 from the posterior tibial artery and 24 from the peroneal artery) were identified preoperatively using the Doppler ultrasonography. Twenty-four flaps were based on tibialis posterior artery perforators and 22 on peroneal artery perforators. Twenty-eight flaps had one perforator on preoperative imaging, and 18 flaps had more than one perforator. Examples of perforators are illustrated in Fig. 1.

The overall average perforator caliber was $1.1 \mathrm{~mm}$. Among the tibial artery perforators, 12 were located in the proximal third, 14 in the middle third, and 12 in the distal third of the leg, with mean calibers of $0.9 \mathrm{~mm}, 1.1 \mathrm{~mm}$, and $1.3 \mathrm{~mm}$, respectively. Among the peroneal artery perforators, six were in the proximal third, nine in the middle third, and 19 in the distal third (mean calibers of $0.8 \mathrm{~mm}, 1.1 \mathrm{~mm}$, and $1.2 \mathrm{~mm}$, respectively).

In two of the flaps that had only one perforator in the preoperative Doppler ultrasonographic analysis, the surgical dissection showed branched vessels immediately superficial to the fascia, assuming the classic shape of a "Y" letter (Fig. 2C).

In two of the other flaps anticipated to have only one perforator, the dissection showed two or three very close perforators at the preoperatively marked point of emergence. The perforators were too close to be distinguished using the Doppler ultrasonography. However, after proceeding with further distal dissection, they converged in a single vessel, with two different shapes: one " $\lambda$ " type (lambda, Fig. 2A) and one "reverse $\psi$ " type (reverse phi, Fig. 2B), respectively. Details on the two converging perforators are summarized in Table 2 , and an anatomical schematic is presented in Fig. 3.

In the 24 single perforator flaps, the planned rotation was achieved without difficulties. The same took place for the two cases of "Y"-shaped perforators; since the pivot point was at the 


\section{Table 1. Details about patients and perforators}

\begin{tabular}{|c|c|c|c|c|c|c|c|}
\hline Patient no. & Sex & Age (yr) & Source artery & $\begin{array}{l}\text { No. of perforator } \\
\text { per flap }\end{array}$ & $\begin{array}{c}\text { Perforator caliber } \\
(\mathrm{mm})\end{array}$ & $\begin{array}{l}\text { Perforator } \\
\text { pattern }\end{array}$ & Zone \\
\hline 1 & $M$ & 47 & Tibial & 1 & 1.4 & Non branching & Proximal \\
\hline 2 & $\mathrm{~F}$ & 37 & Peroneal & 1 & 0.5 & Non branching & Middle \\
\hline 3 & $\mathrm{~F}$ & 53 & Tibial & 2 & 1.4 & Non branching & Distal \\
\hline 4 & $\mathrm{~F}$ & 25 & Tibial & 2 & 1.1 & Non branching & Distal \\
\hline 5 & M & 61 & Tibial & 3 & 1.3 & Non branching & Middle \\
\hline 6 & M & 50 & Tibial & 1 & 1.2 & $Y$ & Proximal \\
\hline 7 & $\mathrm{~F}$ & 61 & Peroneal & 2 & 1.5 & Non branching & Distal \\
\hline 8 & M & 56 & Peroneal & 1 & 1.1 & Reverse $\psi$ & Proximal \\
\hline 9 & M & 57 & Tibial & 3 & 1.7 & Non branching & Middle \\
\hline 10 & M & 38 & Peroneal & 1 & 1.3 & Non branching & Middle \\
\hline 11 & M & 39 & Tibial & 1 & 0.6 & Non branching & Proximal \\
\hline 12 & M & 43 & Tibial & 1 & 0.5 & Non branching & Proximal \\
\hline 13 & M & 50 & Tibial & 2 & 1.0 & Non branching & Middle \\
\hline 14 & M & 56 & Peroneal & 1 & 1.4 & Non branching & Distal \\
\hline 15 & $\mathrm{~F}$ & 40 & Peroneal & 3 & 1.5 & Non branching & Distal \\
\hline 16 & M & 36 & Peroneal & 1 & 1.1 & Non branching & Distal \\
\hline 17 & M & 42 & Peroneal & 2 & 1.2 & Non branching & Distal \\
\hline 18 & $\mathrm{~F}$ & 34 & Tibial & 3 & 0.9 & Non branching & Middle \\
\hline 19 & M & 29 & Peroneal & 1 & 1.3 & Non branching & Distal \\
\hline 20 & M & 53 & Tibial & 1 & 0.6 & Y & Distal \\
\hline 21 & $\mathrm{~F}$ & 27 & Peroneal & 1 & 1.0 & Non branching & Middle \\
\hline 22 & $\mathrm{~F}$ & 36 & Peroneal & 2 & 0.8 & Non branching & Middle \\
\hline 23 & $\mathrm{~F}$ & 47 & Tibial & 1 & 1.2 & Non branching & Middle \\
\hline 24 & $\mathrm{~F}$ & 32 & Peroneal & 3 & 0.7 & Non branching & Proximal \\
\hline 25 & M & 60 & Tibial & 1 & 1.0 & Non branching & Proximal \\
\hline 26 & $\mathrm{~F}$ & 63 & Tibial & 1 & 0.5 & Non branching & Middle \\
\hline 27 & M & 53 & Tibial & 2 & 0.8 & Non branching & Proximal \\
\hline 28 & $\mathrm{~F}$ & 58 & Tibial & 1 & 1.4 & Non branching & Middle \\
\hline 29 & M & 30 & Tibial & 1 & 1.6 & Non branching & Distal \\
\hline 30 & M & 43 & Peroneal & 1 & 0.5 & Non branching & Proximal \\
\hline 31 & M & 51 & Peroneal & 1 & 1.5 & $\lambda$ & Middle \\
\hline 32 & M & 60 & Tibial & 2 & 0.7 & Non branching & Proximal \\
\hline 33 & M & 64 & Tibial & 3 & 1.2 & Non branching & Proximal \\
\hline 34 & M & 48 & Peroneal & 1 & 0.7 & Non branching & Proximal \\
\hline 35 & M & 65 & Peroneal & 2 & 1.6 & Non branching & Middle \\
\hline 36 & M & 42 & Tibial & 2 & 1.7 & Non branching & Distal \\
\hline 37 & $\mathrm{~F}$ & 58 & Peroneal & 1 & 1.3 & Non branching & Distal \\
\hline 38 & $\mathrm{~F}$ & 44 & Tibial & 1 & 1.4 & Non branching & Distal \\
\hline 39 & M & 26 & Peroneal & 1 & 0.6 & Non branching & Distal \\
\hline 40 & M & 59 & Peroneal & 3 & 1.7 & Non branching & Distal \\
\hline 41 & $\mathrm{~F}$ & 61 & Peroneal & 1 & 1.1 & Non branching & Distal \\
\hline 42 & M & 38 & Tibial & 1 & 1.0 & Non branching & Distal \\
\hline 43 & M & 44 & Peroneal & 1 & 1.3 & Non branching & Middle \\
\hline 44 & M & 48 & Tibial & 1 & 1.6 & Non branching & Distal \\
\hline 45 & $\mathrm{~F}$ & 30 & Peroneal & 3 & 1.0 & Non branching & Distal \\
\hline 46 & M & 38 & Tibial & 1 & 1.6 & Non branching & Distal \\
\hline
\end{tabular}

base of the "Y," the arc of rotation was the same as that of a single perforator flap.

In the 18 flaps with multiple perforators, in order to achieve the correct arc of rotation and to avoid excessive kinking, only one perforator had to be preserved. The choice of the most reliable perforator was based on vessel calibre, quality of pulse, and favorability of the position. The other perforators were clipped and cut.

In the two flaps with converging perforators, selective preservation of the more reliable vessel was initially planned, as for the flaps presenting with multiple perforators. After the distal dissection and the discovery of the common branch, though, the 
Table 2. Characteristics of the two converging perforators

\begin{tabular}{|ccccccc|}
\hline Patient no. & Sex & Age $(\mathrm{yr})$ & Source artery & Perforator caliber $(\mathrm{mm})$ & Perforator pattern & Zone \\
\hline 8 & Male & 56 & Peroneal & 1.1 & Reverse $\psi$ & Proximal \\
31 & Male & 51 & Peroneal & 1.5 & $\lambda$ & Middle \\
\hline
\end{tabular}

\section{Fig. 1. Single non-branching perforators}

Intraoperative photos of single perforators. (A) A classic single perforator flap, with a single vessel emerging from the fascia and entering the flap. $(B, C)$ Flaps with more than one perforator (2 and 3, respectively). A normal vascular course is observed in these photos.
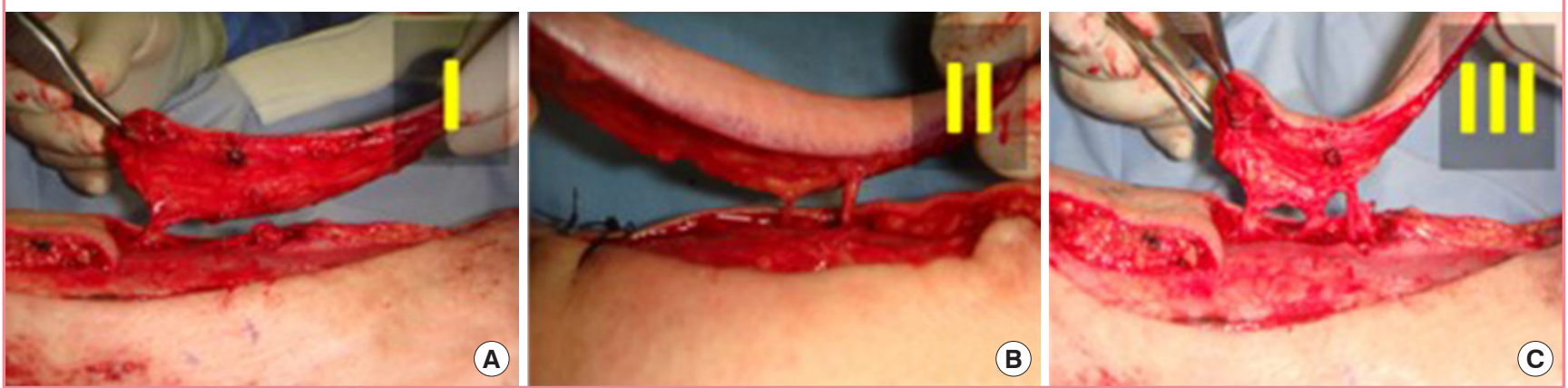

\section{Fig. 2. Suprafascial branching perforators}

This figure illustrates the vascular branching patterns encountered in our series. (A) The 2-in- 1 " $\lambda$ " pattern; at the base are two close perforators, just above the fascia; at the apex, the two perforators converge in a single common branch, entering the flap. (B) The 3-in- 1 "reverse $\psi$ " pattern; three different perforators converge in a single branch. (C) The 1-in-2 "Y" pattern; in contrast to (A) and (B), here a single perforator diverges into two smaller branches. This is considered to be a normal anatomical configuration.
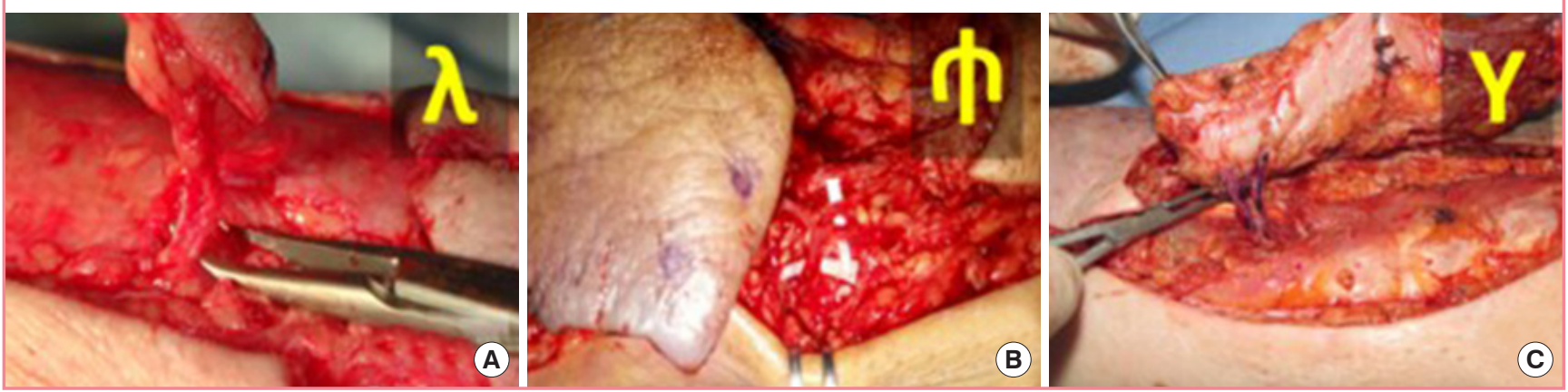

Fig. 3. Anatomical schematic of perforator courses

This schematic shows a simplified portrayal of the relevant anatomy, designating the three planes through which the perforators run. The same anatomical configurations are presented as in Fig. 2.
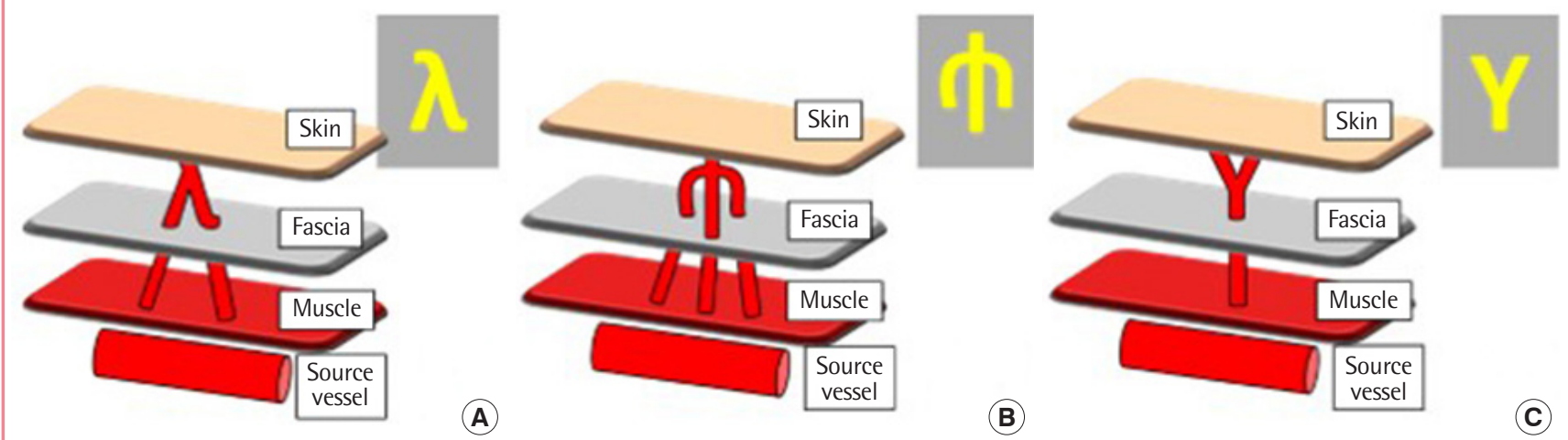
pivot point was changed to the distal single branch, thereby achieving the same pivot as the single perforator flaps.

Similar cases have not been reported in the literature, and we herein describe the first case of convergent suprafascial vascular branches.

\section{DISCUSSION}

Lower leg reconstruction often requires the use of local perforator flaps, based on either the tibialis posterior artery [15-18] or the peroneal artery [8,19-24]. Propeller and advancement flaps for reconstruction of the lower extremity are the most widely used local flaps in the leg, and often constitute the final reconstructive option before a distant free flap is required $[2,25]$.

The Dopplex device is currently a standard modality for preoperative evaluations in perforator flap reconstructive surgery $[9,11,13]$. Doppler ultrasonography is considered to be highly predictive of perforator vessel localization, despite a range of reported false positive and false negative rates $[11,13]$. More specifically, Doppler ultrasonography identifies the point where the vessel perforates the fascia.

Our series shows that a minority of perforators emerged as branched vessels, rather than as single vessels. In these cases, ultrasonography was unable to distinguish single non-branching perforators from branching perforators.

When multiple perforators are encountered during suprafascial dissection, the surgeon must choose the best perforator to achieve the proper rotation of the flap. Vascular branches and parallel close perforators increase the risk of kinking.

Our study shows that very close perforators might converge in a single distal branch. If the dissection is further extended distally along such vessels, the surgeon might not be compelled to choose between perforators. In this way, the entire area of vasculature and the native anatomy are preserved. Through awareness of possible variations in the suprafascial course of perforator vessels, the reconstructive surgeon can rotate a planned flap without further reducing its arterial supply.

\section{NOTES}

\section{Conflict of interest}

No potential conflict of interest relevant to this article was reported.

\section{Ethical approval}

The study was approved by the I.R.C.C.S. San Raffaele Hospital Ethical Committee (approval No. CE-15/int/2020) and was conducted in accordance with the Ethical Principles for Medical
Research Involving Human Subjects, as outlined in the Helsinki Declaration of 1975. Written informed consents were obtained.

\section{Patient consent}

The patients provided written informed consent for the publication and the use of their images.

\section{Author contribution}

Conceptualization: Amendola F, Vaienti L. Data curation: Cottone G, Borelli F, De Francesco F, Zaccaria G. Formal analysis: Amendola F. Methodology: Amendola F. Project administration: Amendola F. Visualization: De Francesco F. Writing original draft: Amendola F. Writing - review \& editing: Amendola F, Vaienti L. Approval of final manuscript: all authors.

\section{ORCID}

Luca Vaienti https://orcid.org/0000-0002-5309-5205 Francesco Amendola https://orcid.org/0000-0002-1410-7568

\section{REFERENCES}

1. Soltanian H, Garcia RM, Hollenbeck ST. Current concepts in lower extremity reconstruction. Plast Reconstr Surg 2015; 136:815e-829e.

2. Bekara F, Herlin C, Somda S, et al. Free versus perforatorpedicled propeller flaps in lower extremity reconstruction: what is the safest coverage? A meta-analysis. Microsurgery 2018;38:109-19.

3. Engel H, Lin CH, Wei FC. Role of microsurgery in lower extremity reconstruction. Plast Reconstr Surg 2011;127 Suppl 1:228S-238S.

4. Bekara F, Herlin C, Mojallal A, et al. A systematic review and meta-analysis of perforator-pedicled propeller flaps in lower extremity defects: identification of risk factors for complications. Plast Reconstr Surg 2016;137:314-31.

5. Blondeel PN, Van Landuyt KH, Monstrey SJ, et al. The "Gent" consensus on perforator flap terminology: preliminary definitions. Plast Reconstr Surg 2003;112:1378-83.

6. Sinna R, Boloorchi A, Mahajan AL, et al. What should define a "perforator flap"? Plast Reconstr Surg 2010;126:225863.

7. Niranjan NS, Price RD, Govilkar P. Fascial feeder and perforator-based V-Y advancement flaps in the reconstruction of lower limb defects. Br J Plast Surg 2000;53:679-89.

8. Gir P, Cheng A, Oni G, et al. Pedicled-perforator (propeller) flaps in lower extremity defects: a systematic review. J Reconstr Microsurg 2012;28:595-601.

9. Feng S, Min P, Grassetti L, et al. A prospective head-to-head 
comparison of color doppler ultrasound and computed tomographic angiography in the preoperative planning of lower extremity perforator flaps. Plast Reconstr Surg 2016;137: 335-47.

10. Panagiotopoulos K, Soucacos PN, Korres DS, et al. Anatomical study and colour Doppler assessment of the skin perforators of the anterior tibial artery and possible clinical applications. J Plast Reconstr Aesthet Surg 2009;62:1524-9.

11. Martin AL, Bissell MB, Al-Dhamin A, et al. Computed tomographic angiography for localization of the cutaneous perforators of the leg. Plast Reconstr Surg 2013;131:792800.

12. Su W, Lu L, Lazzeri D, et al. Contrast-enhanced ultrasound combined with three- dimensional reconstruction in preoperative perforator flap planning. Plast Reconstr Surg 2013; 131:80-93.

13. Miller JR, Potparic Z, Colen LB, et al. The accuracy of duplex ultrasonography in the planning of skin flaps in the lower extremity. Plast Reconstr Surg 1995;95:1221-7.

14. Tang M, Mao Y, Almutairi K, et al. Three-dimensional analysis of perforators of the posterior leg. Plast Reconstr Surg 2009; 123:1729-38.

15. Koshima I, Moriguchi T, Ohta S, et al. The vasculature and clinical application of the posterior tibial perforator-based flap. Plast Reconstr Surg 1992;90:643-9.

16. Drimouras G, Kostopoulos E, Agiannidis C, et al. Redefining vascular anatomy of posterior tibial artery perforators: a cadaveric study and review of the literature. Ann Plast Surg 2016;76:705-12.

17. Hupkens P, Westland PB, Schijns W, et al. Medial lower leg perforators: an anatomical study of their distribution and characteristics. Microsurgery 2017;37:319-26.

18. Vaienti L, Calori GM, Leone F, et al. Posterior tibial artery perforator flaps for coverage of Achilles region defects. Injury 2014;45 Suppl 6:S133-7.

19. Georgescu AV. Propeller perforator flaps in distal lower leg: evolution and clinical applications. Arch Plast Surg 2012;39: 94-105.

20. Ozalp T, Masquelet AC, Begue TC. Septocutaneous perforators of the peroneal artery relative to the fibula: anatomical basis of the use of pedicled fasciocutaneous flap. Surg Radiol Anat 2006;28:54-8.

21. Gacevic M, Milisavljevic M, Novakovic M, et al. Skin vascularisation field by the ascending branch of the peroneal artery ramus perforans. Vojnosanit Pregl 2011;68:575-82.

22. Lykoudis EG, Koutsouris M, Lykissas MG. Vascular anatomy of the integument of the lateral lower leg: an anatomical study focused on cutaneous perforators and their clinical importance. Plast Reconstr Surg 2011;128:188-98.

23. Heitmann C, Khan FN, Levin LS. Vasculature of the peroneal artery: an anatomic study focused on the perforator vessels. J Reconstr Microsurg 2003;19:157-62.

24. Lu TC, Lin CH, Lin CH, et al. Versatility of the pedicled peroneal artery perforator flaps for soft-tissue coverage of the lower leg and foot defects. J Plast Reconstr Aesthet Surg 2011;64:386-93.

25. Innocenti M, Menichini G, Baldrighi C, et al. Are there risk factors for complications of perforator-based propeller flaps for lower-extremity reconstruction? Clin Orthop Relat Res 2014;472:2276-86. 